\title{
Chest percussion, a common yet underutilized art
}

\author{
Neeraj Kumar Gupta ${ }^{1}$, Rohit Kumar ${ }^{1}$, Harsh Vardhan Puri ${ }^{2}$, Nitesh Gupta ${ }^{1}$, Siddharth Raj Yadav ${ }^{1}$, \\ Pranav Ish ${ }^{1}$ \\ ${ }^{1}$ Department of Pulmonary, Critical Care and Sleep Medicine, Vardhaman Mahavir Medical College and Safdarjung \\ Hospital, New Delhi; ${ }^{2}$ Department of Thoracic Surgery, Sir Ganga Ram Hospital, New Delhi, India
}

\section{Dear Editor}

Long years of respiratory medicine practice lets one develop a clinical instinct which certainly aid in diagnostic acumen. A senior professor developed dry episodic cough with some retro-sternal discomfort. He had no fever, pain, dyspnoea or any other constitutional symptoms. Physically very active and an avid sportsman, he continued with patient care. He self-percussed his thorax to look for mediastinum shift, crepitus and liver dullness and found a hyper resonant note in right side of the chest in axillary and inframammary area. The physician promptly got a non-contrast CT Chest (Figure 1 A,B) which confirmed right sided pneumothorax with underlying ruptured bulla. Since it was the first episode with minimal symptoms, a detailed discussion with the surgeon was undertaken regarding the management. In view of evidence of a ruptured bulla, a Video assisted thoracoscopic surgery was considered the best option even though oxygen saturation and hemodynamic stability was remarkable. Two bullae were removed by wedge resection (Figure $1 \mathrm{C}, \mathrm{D}$ ), complete parietal pleura was

Correspondence: Pranav Ish, Assistant Professor, Department of Pulmonary, Critical Care and Sleep Medicine, Vardhaman Mahavir Medical College and Safdarjung Hospital, New Delhi110029, India. Tel. +91.9958356000

E-mail: pranavish2512@gmail.com

Key words: Percussion; self-percussion; early diagnosis.

Conflict of interest: The authors declare that they have no competing interests, and all authors confirm accuracy.

Informed consent: The authors certify that they have obtained all appropriate patient consent forms. In the form the patient has given his consent for his images and other clinical information to be reported in the journal. The patient understand that his name and initials will not be published and due efforts will be made to conceal their identity, but anonymity cannot be guaranteed.

Received for publication: 17 May 2020.

Accepted for publication: 18 June 2020.

${ }^{\circ}$ Copyright: the Author(s), 2020

Licensee PAGEPress, Italy

Monaldi Archives for Chest Disease 2020; 90:1387

doi: 10.4081 /monaldi.2020.1387

This article is distributed under the terms of the Creative Commons Attribution Noncommercial License (by-nc 4.0) which permits any noncommercial use, distribution, and reproduction in any medium, provided the original author(s) and source are credited. removed, and mechanical pleurodesis was done. Two intercostal drainage tubes (apical and basal) with negative suction were put and removed on day 5 post-operatively. The patient (doctor) was discharged with expanded lung and clinically asymptomatic.

The ancient art of percussion in respiratory system examination was first described by Auenbrugger in 1761 [1]. Laennec, in the late $17^{\text {th }}$ century, further developed the percussion notes interpretation and correlated it with auscultation to differentiate emphysema, pleural effusion and pneumothorax [2]. However, even after three decades the art and science of percussion remains grossly underutilized [2]. The stethoscope, a quick Chest X-ray and advanced radiology like CT chest has been the main cause, but we as respiratory physicians must realise that we do not carry
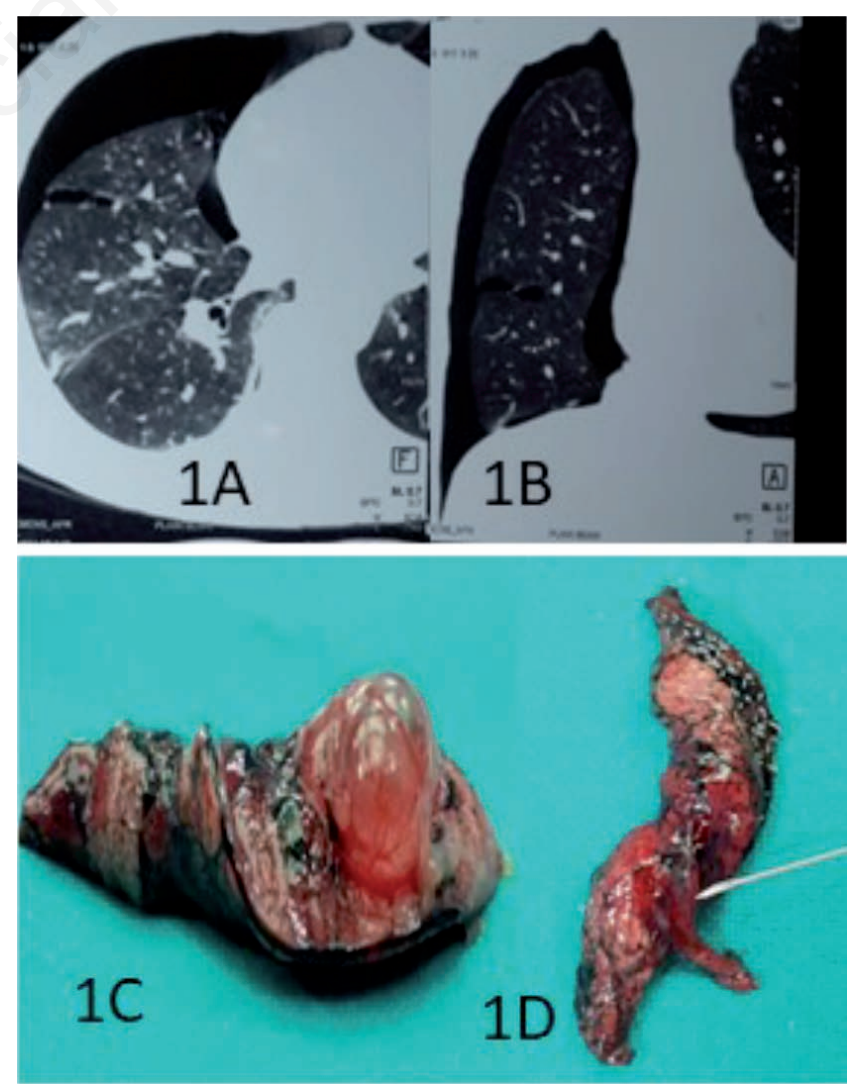

Figure 1. A) Non-contrast CT chest revealing right sided pneumothorax due to a ruptured bulla. B) A coronal view of the pneumothorax showing a ruptured and an intact bulla in the right lung. C) Wedge resection of the intact bulla. D) Wedge resection of the ruptured bulla marked by the pointer. 
our stethoscope in daily activities, but we can always percuss with our bare hands.

Self-percussion has scant mention in literature. It is rarely used in medical school to teach the art of percussion [3] and has a definite role as a part of rehabilitation in bronchiectasis [4,5]. This case highlights the importance of the ancient art of percussion. By teaching percussion and auscultation to the residents, the professor had perfected his clinical skills; thereby utilizing the clinical skill to make an early diagnosis. An early diagnosis allowed for a definitive procedure done within a day from the first symptom.

\section{References}

1. Ellis H. Leopold Auenbrugger, father of the clinical examination of the chest. Br J Hosp Med (Lond) 2009;70:415.

2. Yernault JC, Bohadana AB. Chest percussion. Eur Respir J 1995;8:1756-60.

3. Schneiderman H. Do attending physicians really percuss? Am J Med 1991;91:325-27.

4. Harris A. Listening-touch, affect and the crafting of medical bodies through percussion. Body Soc 2016;22:31-61.

5. Langenderfer B. Alternatives to percussion and postural drainage. A review of mucus clearance therapies: percussion and postural drainage, autogenic drainage, positive expiratory pressure, flutter valve, intrapulmonary percussive ventilation, and high-frequency chest compression with the ThAIRapy Vest. J Cardiopulm Rehabil 1998;18:283-9. 